\title{
The mean metallicity - kinematics relation in high column density MgII absorbers and selection effects in DLA surveys
}

\author{
David A. Turnshek ${ }^{1,2}$, Sandhya M. Rao ${ }^{1}$, Daniel B. Nestor ${ }^{1,3}$, \\ Michèle Belfort-Mihalyi ${ }^{1}$, and Anna M. Quider ${ }^{1}$
}

${ }^{1}$ Department of Physics and Astronomy, University of Pittsburgh, Pittsburgh, PA 15260, USA

${ }^{2}$ Email: turnshek@pitt.edu, ${ }^{3}$ Present address: Department of Astronomy, University of Florida

\begin{abstract}
Sloan Digital Sky Survey (SDSS) quasar spectroscopy is yielding a database of strong low-ionisation MgII absorbers over the redshift interval $0.36<z<2.28$ which is over two orders of magnitude larger than anything previously assembled. Hubble Space Telescope (HST) UV spectroscopy has been used to measure neutral hydrogen column densities for a small subset of them. These data empirically show that MgII absorbers with rest equivalent widths $W_{0}^{\lambda 2796} \geqslant 0.6 \AA$ have a mean neutral hydrogen column density that is roughly constant at $N(H I) \approx 4 \times 10^{20}$ atoms $\mathrm{cm}^{-2}$, with individual systems lying in the damped Ly $\alpha$ (DLA) and sub-DLA regimes. Since the MgII doublets generally exhibit saturation, the $W_{0}^{\lambda 2796}$ values are an indication of the absorbers' velocity spreads. Thus, we can study neutral-gas-phase metallicities as a function of kinematics by forming SDSS composite spectra and measuring weak unsaturated metal lines that form in neutral gas (e.g., CrII, FeII, MnII, SiII, ZnII) as a function of $W_{0}^{\lambda 2796}$. We use this method on SDSS composite spectra to show how metallicity and kinematics are positively correlated for large- $N(H I)$ absorbers, including trends related to dust depletion and the enhancement of $\alpha$-elements. We also discuss the need to account for selection effects in DLA surveys, and we make inferences about models for DLA absorption and their contribution to cosmic star formation.
\end{abstract}

\section{The properties of high column density absorbers}

Rao (2005; this volume) has reviewed the statistics of DLAs, with some emphasis on results at redshifts $z<1.65$ based on HST UV spectroscopy. See also Rao, Turnshek \& Nestor (2005a, hereafter RTN2005). It is widely believed that the classical DLAs with $N(H I) \geqslant 2 \times 10^{20}$ atoms $\mathrm{cm}^{-2}$ track the neutral gas component of the Universe at $z<4$ (but see $\S 4$ ). The measurements indicate that the neutral gas mass in DLAs is approximately constant from $z \approx 4$ to $z \approx 0.5$, but drops by a factor of $\approx 2$ from $z \approx 0.5$ to $z=0$. Also, relative to no-evolution, the product of gas cross-section and co-moving number density of absorbers drops by a factor of $\approx 2$ from $z \approx 4$ to $z \approx 2$, but then follows the no-evolution curve from $z \approx 2$ to $z=0$ ( $h=0.7, \Omega=0.3, \Lambda=0.7)$. At $z<1$, where searches for galaxies associated with DLAs have been reasonably successful, a mix of galaxy types have been found, e.g. dwarfs, low surface brightness (LSB) galaxies, luminous spirals, etc. (see Rao et al. 2003 and references therein). In addition, the mean column-density-weighted metallicity of the DLAs increases from $[\mathrm{X} / \mathrm{H}] \approx-1.6$ at $z \approx 4$ to $[\mathrm{X} / \mathrm{H}] \approx-0.8$ at $z \approx 1$ where $\mathrm{X}$ is $\mathrm{Zn}, \mathrm{Fe}, \mathrm{O}$, or Si (e.g. see Rao et al. 2005b and references therein). Aside from these trends, we also previously reported a positive correlation between mean metallicity and kinematics in high- $N(H I)$ systems (Nestor et al. 2003); one of the aims of this contribution is to discuss more recent work on the details of this relationship ( $(2)$. We emphasise that while substantial observational progress on DLAs has been made over the last five years (this volume), properly connecting these 
results to scenarios for galaxy and structure formation will require careful consideration of selection effects; we will consider several applications to illustrate this ( $\S 3)$. We will also discuss the implications of our results and other recent work $(\S 4)$.

\section{The metallicity - kinematics relation in large- $\mathrm{N}(\mathrm{HI})$ absorbers}

\subsection{Formation of SDSS composite spectra vs. MgII rest equivalent width}

The MgII $\lambda \lambda 2796,2803$ absorbers visible in SDSS quasar spectra cover the redshift interval $0.36<z<2.28$. The number of systems in the completed SDSS will be well over two orders of magnitude larger than anything previously assembled. As is now well established, the DLAs are traced by a subset of the strong MgII absorbers, and track the bulk of the HI gas mass that has so far been observed in the Universe. In the study described here we are interested in using the MgII absorbers to investigate the mean relationship between metallicity and velocity spread in large- $N(H I)$ absorbers. The idea is as follows. HST UV spectroscopy has been used to measure HI column densities for a small subset of the SDSS MgII systems, each of which has a known rest equivalent width (REW) $W_{0}^{\lambda 2796}$ (Rao 2005, this volume; RTN2005). From these data we know empirically that for MgII absorption lines with $0.3 \leqslant W_{0}^{\lambda 2796}<0.6 \AA$ the mean HI column density is $N(H I) \approx 10^{19}$ atoms $\mathrm{cm}^{-2}$; for $W_{0}^{\lambda 2796} \geqslant 0.6 \AA$ it is roughly constant at $N(H I) \approx 4 \times 10^{20}$ atoms $\mathrm{cm}^{-2}$ with individual $N(H I)$ values spanning about 3 orders of magnitude and falling within both the sub-DLA and DLA regimes (typically $10^{18.7}<N(H I)<10^{21.7}$ atoms $\mathrm{cm}^{-2}$ ). However, the MgII doublets generally exhibit saturation, so the $W_{0}^{\lambda 2796}$ values are an indication of the absorbers' velocity spreads. Thus, we can study neutral-gas-phase metallicities as a function of kinematics by forming SDSS composite spectra and measuring weak unsaturated metal lines as a function of $W_{0}^{\lambda 2796}$. To accomplish this we followed a method similar to the one described by Nestor et al. (2003), who studied the neutral gas phase element abundances of Zn and Cr. The MgII absorbers incorporated in this analysis were identified in SDSS quasar spectra up to DR3 using the Nestor, Turnshek \& Rao (2005a) selection criteria. Nearly 6000 absorption systems were used to form composite spectra for different intervals of $W_{0}^{\lambda 2796}$ in regions covering $\mathrm{MgII}$ and $\mathrm{MgI}$, and the low oscillator strength metal lines due to MnII, FeII, ZnII, CrII, and SiII. These composites approximately covered the redshift interval $1<z<2$, as dictated by the rest wavelengths of the metal lines and the short wavelength cutoff of SDSS at $\approx 3800 \AA$. As examples, the left side of Fig. 1 shows the composites covering the ZnII and CrII region, while the right side shows the composites for the MgII and MgI region.

\subsection{Correlation between mean metallicity and velocity spread}

Measurements of weak metal lines due to MnII, FeII, ZnII, CrII, and SiII in the composite spectra resulted in the mean element abundance determinations graphically illustrated in Fig. 2. In converting the measured REWs to metal-line column densities, saturation effects in the weak lines were assumed to be unimportant. This is consistent with the finding that our results are robust and independent of inclusion of the handful of cases where these metal lines are seen in individual SDSS spectra. In addition, possible ionisation corrections were not made; thus the results incorporate the assumption that singly ionised states of $\mathrm{Mn}, \mathrm{Fe}, \mathrm{Zn}, \mathrm{Cr}$, and $\mathrm{Si}$ are present in the neutral regions that contribute to the measured mean HI column density of $N(H I) \approx 4 \times 10^{20}$ atoms $\mathrm{cm}^{-2}$ at $W_{0}^{\lambda 2796} \geqslant 0.6 \AA$. For Fig. 2 we transformed $W_{0}^{\lambda 2796}$ in units of $\AA$ (see Fig. 1 ) to $\Delta \mathrm{V}_{R E W}=c\left(W_{0}^{\lambda 2796} / 2796\right)$ in units of $\mathrm{km} \mathrm{s}^{-1}$, since this more explicitly makes the point that the $\lambda 2796$ line is saturated and that $W_{0}^{\lambda 2796}$ is most appropriately indicative of a 

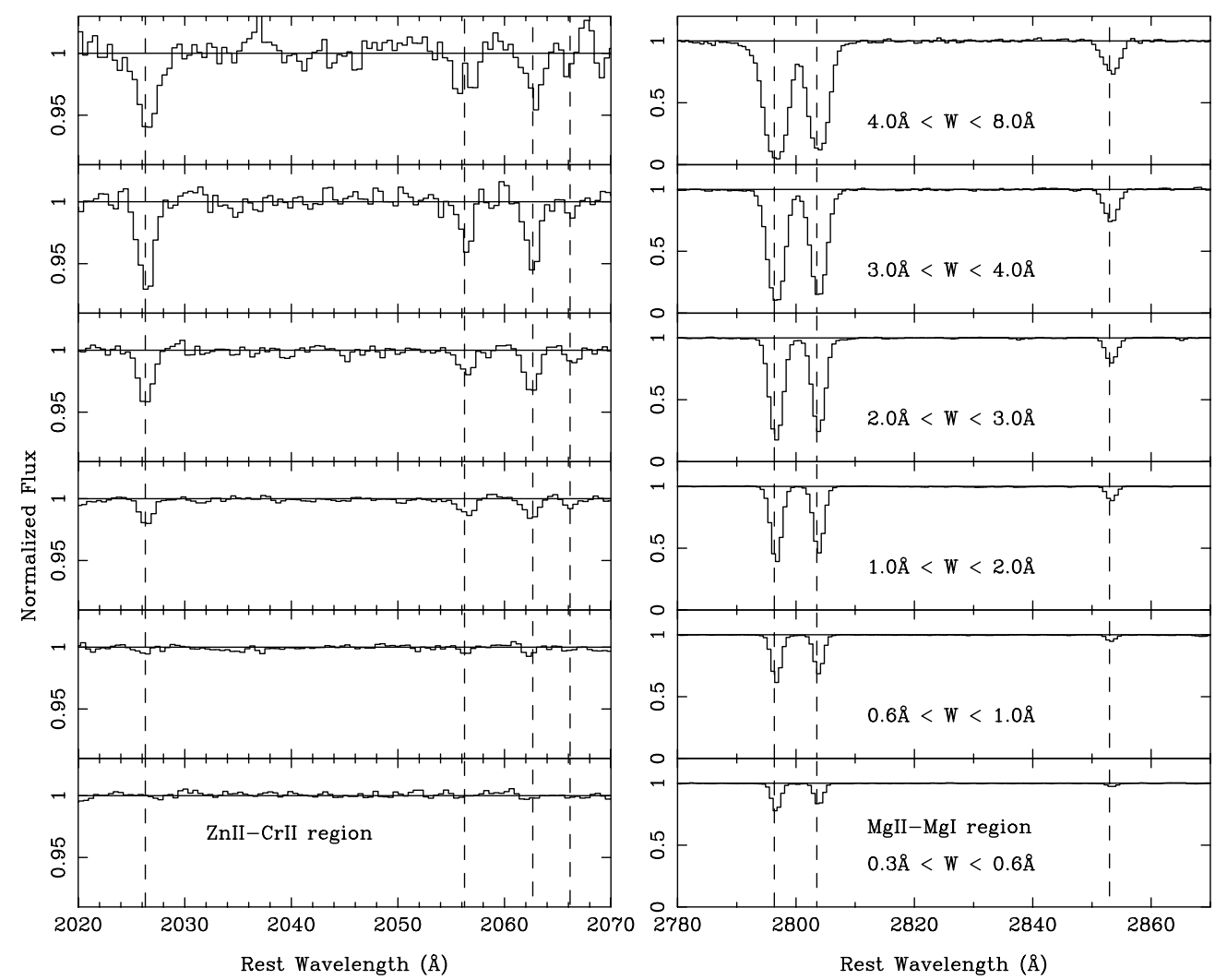

Figure 1. The left side shows the flux-normalised $\mathrm{ZnII}$ and $\mathrm{CrII}$ region of the composite spectra, while the right side shows the corresponding MgII and MgI region. The $N(H I)$ value is constant at $\approx 4 \times 10^{20}$ atoms $\mathrm{cm}^{-2}$ in all of the composite spectra except for the bottom most panels where $N(H I) \approx 10^{19}$ atoms $\mathrm{cm}^{-2}$. $W_{0}^{\lambda 2796}$ increases from bottom to top. The fact that the MgII doublet is saturated indicates that increases in $W_{0}^{\lambda 2796}$ are primarily due to larger velocity spreads in the metal-line absorption. At the same time, the much weaker REWs of the $\mathrm{ZnII}$ and CrII metal lines (note the scale on the normalised flux) indicate that these lines are unsaturated, hence the increase in their REWs from bottom to top is due to an increase in metallicity.

velocity width or spread (although $\Delta \mathrm{V}_{R E W}$ is not the Doppler parameter and $W_{0}^{\lambda 2796}$ does increase as the log of the product of column density and oscillator strength in this regime, so these velocity values need to be interpreted carefully).

We see that the overall level of metal enrichment in strong MgII absorption systems can be separated out simply on the basis of $W_{0}^{\lambda 2796} \equiv \Delta \mathrm{V}_{R E W}$, i.e. a positive relationship between mean metallicity and kinematics. However, within this overall relationship, several notable trends are present. We discuss these separately below.

(1) Fe, Zn, and Cr. These elements are often assumed to track the Fe-peak elements and to have a common origin. Results indicate that Zn suffers little depletion in DLA absorbers, so throughout we will assume that $\mathrm{Zn}$ is not depleted, although some $\mathrm{Zn}$ depletion might be expected at the highest metallicities. Measurements of the FeII, ZnII, and CrII lines at the lowest velocity spreads are uniformly consistent with a mean abundance of $[\mathrm{X} / \mathrm{H}] \approx-2.0(1 \%$ Solar) and therefore Fe and $\mathrm{Cr}$ show no evidence for depletion. However, the ZnII measurement at the highest velocity spreads yields a mean abundance of $[\mathrm{Zn} / \mathrm{H}] \approx-0.4$ (40\% Solar), and the more depleted elements of $\mathrm{Fe}$ and $\mathrm{Cr}$ have abundances of $[\mathrm{Fe} / \mathrm{H}] \approx-1.1$ (i.e. $[\mathrm{Fe} / \mathrm{Zn}] \approx-0.7$, or $\mathrm{Fe}$ is at least $\approx 80 \%$ depleted) 


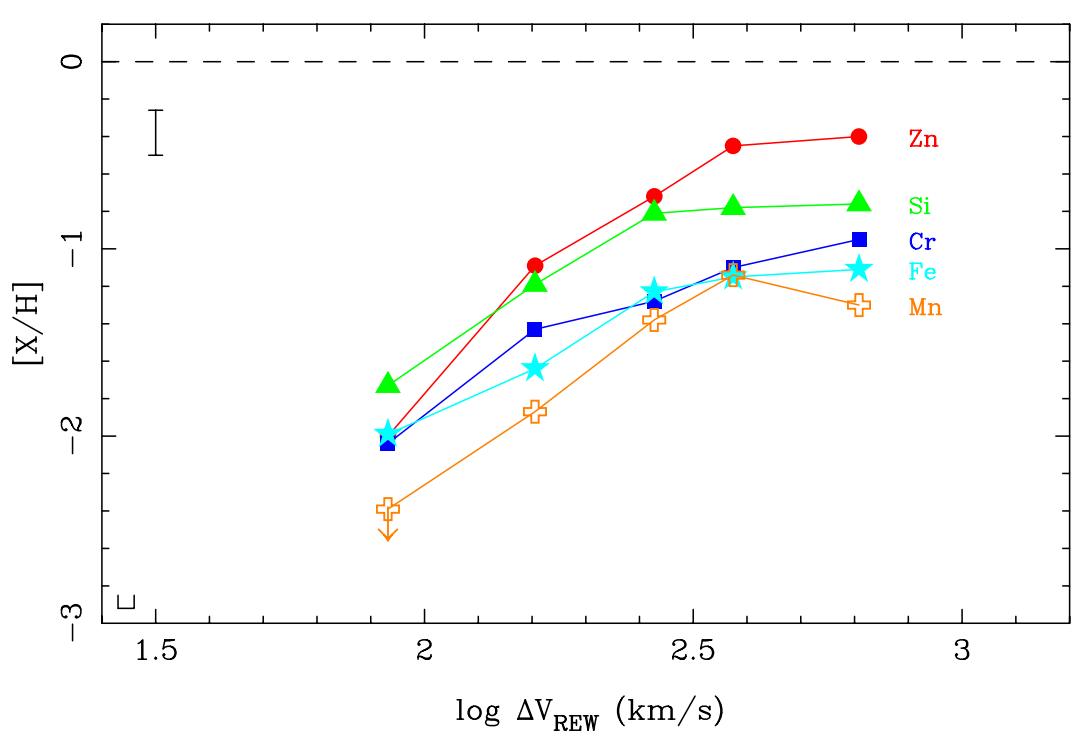

Figure 2. The metallicity of $\mathrm{Zn}, \mathrm{Si}, \mathrm{Cr}, \mathrm{Fe}$, and $\mathrm{Mn}$ as a function of $W_{0}^{\lambda 2796}$ in velocity units, $\Delta \mathrm{V}_{R E W}$, which is roughly correlated with the velocity spread of the absorption. As discussed in the text, at small REW velocity the metallicity is low, there is little evidence for depletion on to grains, the Mn abundance is significantly lower than the other Fe-peak elements, and the $\alpha$-element $\mathrm{Si}$ is enhanced. At large REW velocity there is significant evidence for depletion on to grains. The redshift interval covered by these measurements generally lie in the range $1<z<2$.

and $[\mathrm{Cr} / \mathrm{H}] \approx-0.95$ (i.e. $[\mathrm{Cr} / \mathrm{Zn}] \approx-0.55$, or $\mathrm{Cr}$ is at least $\approx 72 \%$ depleted). The mean metallicity - kinematics relation for $\mathrm{Zn}$ in strong $\mathrm{MgII}$ absorbers can be parameterised as $[\mathrm{Zn} / \mathrm{H}]=-0.4-0.0043 e^{\left[\left(6-W_{0}^{\lambda 2796}\right) / 0.88\right]}$, where $0.6 \AA \leqslant W_{0}^{\lambda 2796}<6.0 \AA$. Thus, there is a clear trend showing the overall increase in mean metallicity with increasing velocity spread $\left(W_{0}^{\lambda 2796}\right)$; and depletion increases with increasing mean metallicity, although it should be pointed out that the nucleosynthetic processes which lead to the formation of Zn are still debated (e.g. Umeda \& Nomoto 2002).

(2) Mn. The Fe-peak element Mn displays a somewhat different behaviour. We note that the work discussed by Savage \& Sembach (1996) indicates that Mn and Fe are similarly depleted in warm Galactic halo clouds $([\mathrm{Mn} / \mathrm{Fe}] \approx 0)$, but that Fe is significantly more depleted than $\mathrm{Mn}$ in cool Galactic disk clouds $([\mathrm{Mn} / \mathrm{Fe}] \approx+0.8)$. Our results for the strong $\mathrm{MgII}$ systems show that $[\mathrm{Mn} / \mathrm{Fe}] \approx-0.1$ at the highest velocity spreads when $[\mathrm{Zn} / \mathrm{H}] \approx-0.4$ and that $[\mathrm{Mn} / \mathrm{Fe}]<-0.4$ at the lowest velocity spreads when $[\mathrm{Zn} / \mathrm{H}] \approx-2$. This trend of lower relative $\mathrm{Mn}$ abundance with decreasing metallicity is consistent with individual DLA measurements reported in Fig. 6 of Dessauges-Zavadsky, Prochaska \& D'Odorico (2002), and is also roughly consistent with Mn measurements for Galactic stars (no depletion). Mn is produced mostly by SNeIa, so a common qualitative interpretation of the low $[\mathrm{Mn} / \mathrm{Fe}]$ values in low-metallicity DLAs is that the gas is in a relatively early stage of chemical evolution, and thus has only undergone significant enrichment by SNeII (e.g. Lu et al. 1996).

(3) Si. The $\alpha$-element Si shows interesting trends relative to (assumed) undepleted $\mathrm{Zn}$. At the highest velocity spreads when $[\mathrm{Zn} / \mathrm{H}] \approx-0.4$ we find $[\mathrm{Si} / \mathrm{Zn}] \approx-0.35$, which is likely representative of some Si depletion, but empirically $\mathrm{Si}$ is likely less depleted than Fe (Savage \& Sembach 1996), and this is consistent with our measured value of [Fe/Zn] $\approx-0.7$. At the lowest velocity spreads when $[\mathrm{Zn} / \mathrm{H}] \approx-2$ we find $[\mathrm{Si} / \mathrm{Zn}] \approx+0.25$ and 
$[\mathrm{Fe} / \mathrm{Zn}] \approx 0$. Thus, our results indicate that the $\alpha$-element $\mathrm{Si}$ is enhanced at these low metallicities. This is generally consistent with the well established result that very metalpoor Galactic halo stars show enhanced levels of $\alpha$-elements (e.g. McWilliam 1997). Once again, the results are qualitatively explained by having the low metallicity gas being in a relatively early stage of chemical evolution, without significant enrichment by SNeIa. However, the enhancement of Si in metal poor Galactic stars (e.g. McWilliam 1997) appears to be somewhat more extreme than what we find for our strong MgII sample.

In general, altering the details of the initial mass function (IMF), the star formation rate (SFR), and mixing mechanisms will affect the abundance trends present in gas with cosmic time. For example, in the case described above one might infer that Si at $1<z<2$ is less enhanced in our sample at low metallicity (in comparison to similarly low metallicities in present-day Galactic stars) because of comparatively lower SFRs. Indeed, Si enhancement at low metallicity in our Galaxy is smaller at larger galactocentric distance for fixed metallicity, and this can be attributed to a lower SFR at larger galactocentric distance (Edvardsson et al. 1993).

An interesting exercise is to compare the mean metallicity - kinematics relation derived from composite spectra (Fig. 2) to individual measurements of DLA metallicities from high-resolution spectroscopy (Fig. 3). The individual metallicities shown in Fig. 3 correspond to redshifts $1.78<z<4.22$, while the mean metallicities shown in Fig. 2 correspond to $1<z<2$. Of course, comparison of the two figures is complicated not only by the redshift difference, but also by the fact that one result incorporates all strong MgII absorbers while the other is limited to DLAs. The metallicities used in Fig. 3 are generally taken from tabulations by Prochaska and collaborators (e.g. references given in Prochaska et al. 2003), but the $\Delta \mathrm{V}$ values are representative of the velocity extent over which low-ionisation absorption is clearly visible (not the velocity interval containing $90 \%$ of the gas, which was kindly provided to us for comparison by Jason Prochaska). In either case we find that individual DLA measurements show a trend of increasing metallicity with increasing velocity spread, but the slope becomes flatter when the velocity interval containing $90 \%$ of the gas is used. This trend is also seen in the high-resolution ESO VLT-UVES data presented by Ledoux et al. (2005) at this colloquium.

The results for strong MgII absorbers described above represent gas-phase abundance trends averaged over all neutral hydrogen clouds in all types of absorbing galaxies for a large range of inclination angles and galactocentric distances as sampled by SDSS quasar sight-lines. We suspect that the lower metallicity sight-lines will preferentially correspond to the progenitors of present-day dwarfs, LSB galaxies, and the outer regions of luminous galaxies, while the higher metallicity sight-lines will primarily sample the more chemically evolved regions of more luminous galaxies, often at lower impact parameters. Thus, the observed relationship between mean metallicity and kinematics provides an empirical constraint on the kinematic and/or dynamic evolution of gaseous regions in galaxies (e.g. disk, halo, and protogalactic merging components). It is natural to think that the more chemically evolved gaseous regions also acquire more mechanical energy (e.g. from gravitational processes and SN explosions) which results in these regions exhibiting greater velocity spreads on average.

\section{Selection effects in galaxy surveys}

\subsection{Gas cross-section selection vs. brightness/colour selection}

The most common type of galaxy surveys are based on multi-colour imaging. Since these are magnitude-limited surveys, objects such as dwarf and LSB galaxies have a tendency 


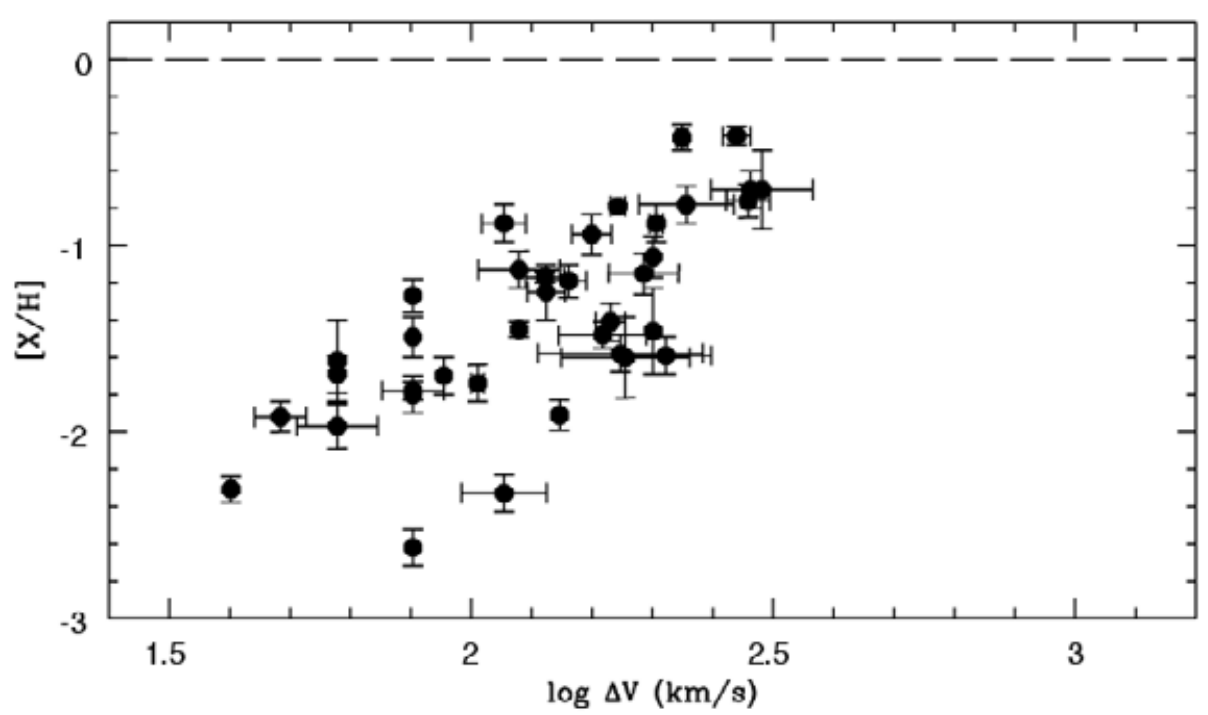

Figure 3. The metallicity - kinematics relation inferred from a subset of DLAs for which high-resolution Keck spectroscopy is readily available (e.g. references given in Prochaska et al. 2003). These DLA absorbers are generally at higher redshift, $1.78<z<4.22$, in comparison to the redshift range covered in the SDSS composite spectra. The $\Delta \mathrm{V}$ parameters, estimated visually from published high-resolution spectra, are the velocity intervals over which low-ionisation metal-line absorption is clearly present. There is a positive correlation between metallicity and $\Delta \mathrm{V}$, consistent with that seen in the SDSS composite spectra.

to be missed, especially with increasing redshift. Alternatively, quasar absorption line observations can be used to conduct completely different types of galaxy surveys. Those which rely on the MgII doublet and/or DLA line identifications are galaxy surveys based on gas-cross-section selection. Yet surveys which use strong MgII selection have a very different physical basis than those which use blind DLA selection.

If reddening/dimming due to dust and amplification due to gravitational lensing were negligible in all DLA systems (but see Ménard et al. 2005), a blind DLA survey yielding a compilation of results as a function of $N(H I)$ would be the most straight-forward way to perform a survey for galaxies based on HI gas cross-section. However, the incidence of DLAs is small enough, especially at low redshift, that we are forced to use the absence of low-ionisation metal lines (e.g. the otherwise easily-detected MgII doublet) as a means to eliminate sight-lines which would have been observed with negative results in any blind DLA survey. As discussed in $\S 2, W_{0}^{\lambda 2796}$ is the most important indicator, since there is little chance of encountering a DLA unless $W_{0}^{\lambda 2796} \geqslant 0.6 \AA$ (Rao \& Turnshek 2000; RTN2005; Rao 2005, this volume). This threshold value, $W_{0}^{\lambda 2796} \equiv \Delta \mathrm{V}_{R E W} \geqslant 65 \mathrm{~km}$ $\mathrm{s}^{-1}$ in velocity units, is the physical basis for MgII-selected surveys and it indicates that DLAs reside in environments that have some minimum value for the velocity spread. Fortunately, high to moderate quality SDSS quasar spectroscopy permits the detection of MgII doublets at this threshold.

At the same time, it is important to recognise that the properties of a MgII-selected sample (e.g. the incidence of DLAs in such a sample) will be biased unless the observed sample's $W_{0}^{\lambda 2796}$ distribution matches the true $W_{0}^{\lambda 2796}$ distribution. The true $W_{0}^{\lambda 2796}$ distribution has recently been determined using SDSS data (Nestor et al. 2005a; Figs. 1 and 2 from Nestor, Turnshek \& Rao 2005b, this volume), so it is now possible to assess 
any mis-match between the observed and true distribution and correct for it if necessary. Below we describe how MgII selection may bias the properties of a sample.

\subsection{Examples: incidence, $N(H I)$ distribution, metallicities, DLA galaxies}

The criteria adopted in a MgII-selected DLA survey may affect the properties of the resulting DLA absorber sample in terms of: (1) the incidence of DLAs per unit redshift, (2) the $N(H I)$ column density distribution of identified DLAs, (3) the resulting DLA metallicities, and most likely (4) the properties of identified DLA galaxies. We briefly discuss each of these effects below.

(1) Dependence of the DLA incidence on $\boldsymbol{W}_{\mathbf{0}}^{\boldsymbol{\lambda 2 7 9 6}}$. As seen in Fig. 4 of Rao (2005, this volume; also RTN2005), the fraction of MgII absorbers which are DLAs with $N(H I) \geqslant 2 \times 10^{20}$ atoms $\mathrm{cm}^{-2}$ depends on $W_{0}^{\lambda 2796}$, rising from a fraction near $16 \%$ just above the threshold value of $W_{0}^{\lambda 2796}=0.6 \AA$ to about $65 \%$ at the highest values near $W_{0}^{\lambda 2796}=3 \AA$. At present these should be considered approximate fractions, since the REW of FeII $\lambda 2600$ can be used (and has been used) to increase the probability of discovering a DLA, however this is a good assessment of the fractions to first order. Thus, unless the observed sample's $W_{0}^{\lambda 2796}$ distribution matches the true distribution, a bias will be introduced into the determination of the DLA incidence. The methods used in RTN2005 account for these differences when calculating the DLA incidence.

(2) Possible dependence of the DLA N(HI) distribution on $W_{0}^{\lambda 2796}$. The degree to which $N(H I)$ may be biased by $W_{0}^{\lambda 2796}$ can be seen by examining the right panel in Fig. 4 of Rao (2005, this volume; also RTN2005). The mean HI column density of identified DLAs is $N(H I) \approx 2.5 \times 10^{21}$ atoms $\mathrm{cm}^{-2}$ when $0.6 \AA \leqslant W_{0}^{\lambda 2796}<1.2 \AA$, but it seems to decrease by a factor of $\approx 4$ at $W_{0}^{\lambda 2796} \approx 3 \AA$. However, inspection of Fig. 2 of Rao (2005, this volume) suggests that this trend is not particularly tight nor is it well established for DLAs by themselves. On the other hand, if one considers all the points in Fig. 2 of Rao (2005), it is clear that the $N(H I)$ distribution changes for different $W_{0}^{\lambda 2796}$ intervals. It is interesting that in MgII-selected surveys for DLAs, the determination of the cosmological mass density of neutral gas, $\Omega_{D L A}$, has (so far) not revealed any dependency on $W_{0}^{\lambda 2796}$ selection. This is because the increased probability (by a factor of $\approx 4$ ) of finding a DLA at the largest $W_{0}^{\lambda 2796}$ values is approximately compensated for by the corresponding decrease in mean HI column density (by a factor of $\approx 4$ ) at the largest $W_{0}^{\lambda 2796}$ values. It is worth pointing out that although the MgII selection criteria which have been employed in low-redshift DLA surveys lead to reasonably complete samples of DLAs, incompleteness must set in at HI column densities in the sub-DLA regime, because systems with $W_{0}^{\lambda 2796}<0.3 \AA$ can have sub-DLA HI column densities. Therefore, only the $N(H I)$ distribution in the DLA regime can be reliably considered with available data.

(3) Dependence of mean metallicity on $\boldsymbol{W}_{\mathbf{0}}^{\boldsymbol{\lambda 2 7 9 6}}$. The analysis described in $\S 2$ demonstrated that the mean metallicity of a MgII-selected sample is a strong function of $W_{0}^{\lambda 2796}$. Although the mean HI column densities of the binned samples were in the DLA regime and constant at $N(H I) \approx 4 \times 10^{20}$ atoms $\mathrm{cm}^{-2}$, most of the individual absorbers are not DLAs. Indeed, above we noted that only $\approx 16 \%$ of the sample are DLAs just above the threshold value of $W_{0}^{\lambda 2796}=0.6 \AA$, and $\approx 65 \%$ are DLAs near $W_{0}^{\lambda 2796}=3 \AA$. Therefore, the results of our metallicity determinations in $\S 2$ apply to strong MgII absorbers, not just DLAs. Of course, it should be kept in mind that in a fixed $W_{0}^{\lambda 2796}$ interval we are sampling a range of $N(H I)$ values, and the sampled systems undoubtedly exhibit a range of metallicities. Thus, a significant amount of detail remains to be discovered through future work. In the end, a reliable determination of the statistical details of cosmic metallicities will depend on unbiased sampling of the $W_{0}^{\lambda 2796}$ 
distribution, or making a correction for biased sampling. The extent to which such biases may have affected existing results (e.g. Prochaska et al. 2003; Rao et al. 2005b) is unclear.

(4) Possible dependence of DLA galaxy type on $\boldsymbol{W}_{0}^{\boldsymbol{\lambda 2 7 9 6}}$. Finally, given the various biases discussed above, it is of interest to consider how an observed sample's $W_{0}^{\lambda 2796}$ distribution may bias the results of searches for DLA galaxies. Significant statistics have not yet been compiled on this issue. Inspection of the results on DLA galaxy identifications (Rao et al. 2003) shows no strong correlations with $W_{0}^{\lambda 2796}$, but sample sizes are still small. Nestor et al. (2005b; this volume) have presented some preliminary evidence which hints at the possibility that very luminous galaxies preferentially lie along the sight-lines to ultra-strong MgII absorbers. In any case, the issue of how MgII selection methods bias the type, luminosity, and impact parameter of identified absorbing galaxies needs to be investigated. Owing to the correlation between mean metallicity and velocity spread (§2.2), some bias is expected. Thus, this inadvertent bias should be accounted for when evaluating, for example, the true frequency of galaxy morphologies, luminosities, and impact parameters that give rise to DLA absorbers.

\section{Implications}

We have presented the results of an analysis which reveals a positive correlation between mean metallicity and gaseous velocity spread in strong MgII absorbers. We have also speculated that this relationship is predominantly due to the low-metallicity, low-velocity-spread sight-lines more preferentially sampling the progenitors of dwarfs, LSB galaxies, and the outer regions of luminous galaxies (primarily because sight-lines through such regions are expected to have low metallicity); whereas the very rare highermetallicity, higher-velocity-spread sight-lines should sample the more chemically evolved regions associated with more luminous galaxies. Rare sight-lines which intercept Lyman break galaxies (LBGs), which can exhibit high metallicity and large velocity spreads in the low-ionisation ISM lines seen in their spectra (e.g. Shapley et al. 2003) may be examples of this latter case. SNeII explosions may drive large bubbles of hot gas out of LBGs and entrain neutral clouds which give rise to DLAs in the flow. But the $W_{0}^{\lambda 2796}$ distribution and the fraction of DLAs as a function of $W_{0}^{\lambda 2796}$ indicates that such situations are rare, happening $<20 \%$ of the time, and therefore are not usually responsible for the DLAs.

An important empirical result which needs to be understood, and which is perhaps more fundamental to explaining the bulk of the DLAs, is why $W_{0}^{\lambda 2796} \equiv \Delta \mathrm{V}_{R E W} \geqslant 65$ $\mathrm{km} \mathrm{s}^{-1}$ is required to find a DLA. The model put forth by Mo, Mao \& White (1998) may provide the beginnings of a scenario to explain this. They studied the formation of galactic disks in cold dark matter (CDM) hierarchical models of structure formation. In their model the bulk of DLAs are produced by centrifugally supported disks within dark matter (DM) halos. The large angular momentum would make these disks more extended and lower in surface brightness (i.e. lower SFRs) in comparison to a low-angularmomentum spheroid that would be more compact and have higher surface brightness (i.e. higher SFRs). Mo, Mao \& White (1999) suggest that such spheroids may correspond to LBGs. Mo et al. (1998) calculate expected impact parameters for DLA disks for various circular velocities that might be required for disk stability. Interestingly, their model predictions are consistent with observed impact parameters of low-redshift DLA galaxies (e.g. compare Fig. 12 in Mo et al. 1998 with Fig. 10 in Rao et al. 2003) when circular velocities of $v_{\text {cir }}>100 \mathrm{~km} \mathrm{~s}^{-1}$ are taken as a requirement for stable disks. Although the details of such a model may need to be modified, one can envision that this type of effect, typically coupled with a reduction in sight-line velocity spread due to inclination 
effects, may largely be responsible for the fact that $W_{0}^{\lambda 2796} \equiv \Delta V_{R E W} \geqslant 65 \mathrm{~km} \mathrm{~s}^{-1}$ is required for a sight-line to encounter a DLA. Mo et al. $(1998,1999)$ comment that various other mechanisms may also be important, and this may depend on the redshift regime. Merging systems of protogalactic clumps (Haehnelt, Steinmetz \& Rauch 1998) and nonequilibrium disks in low-mass DM halos may also contribute to the DLAs. As noted above, LBGs may also from time to time be associated with DLAs, but at much lower incidence since their low angular momentum makes them much less extended. Within all of these possibilities there may not be significant total gas cross-section to generally give rise to a DLA component below the line-of-sight threshold of $W_{0}^{\lambda 2796} \equiv \Delta V_{R E W} \approx 65 \mathrm{~km} \mathrm{~s}^{-1}$. This is an important constraint on future CDM simulations that seek to explain DLAs.

The possibility that some types of regions in the Universe may have too small an interception probability (i.e. gas cross-section times co-moving number density of that type of region) to be easily identified as DLAs in MgII or blind DLA searches is an important issue for another reason. Hopkins, Rao \& Turnshek (2005; also Rao 2005, this volume) have discussed the question of whether the observed population of DLAs can account for the observed cosmic SFR from low to high redshift. By applying the Kennicutt (1998) formulation of the Schmidt law to the properties of the currently observed population of DLAs they find that the DLAs under-predict the cosmic SFR inferred from the luminosity density of high-redshift galaxies (see Fig. 12 in Rao 2005, this volume). An even larger discrepancy occurs when one compares the DLA metallicities to the metallicities expected on the basis of the cosmic SFR (see Fig. 13 in Rao 2005, this volume). One way to avoid this discrepancy is to postulate that the MgII and blind DLA surveys are not yet large enough to include absorbers with very small individual cross-sections that nevertheless may dominate the cosmic SFR and be the main reservoirs for the metals.

Indeed, these star forming regions will be rich in molecular clouds, but the HI column densities along sight-lines passing through them will still lie in the DLA regime. Of course molecular clouds, not HI clouds, most directly provide the fuel for star formation. Kennicutt (1998) points out that in normal disks star formation generally takes place in regions that contain $1-100 \mathrm{M}_{\odot} \mathrm{pc}^{-2}$ (i.e. $\approx 10^{20}-10^{22}$ atoms or molecules $\mathrm{cm}^{-2}$ ), whereas the more rare (and smaller) star burst regions contain $10^{2}-10^{5} \mathrm{M}_{\odot} \mathrm{pc}^{-2}$ (i.e. $\approx 10^{22}-10^{25}$ atoms or molecules $\mathrm{cm}^{-2}$ ). It therefore seems reasonable to conclude that most of the neutral and molecular gas mass has so far been missed in DLA surveys, and this seems consistent with the fact that molecules are rarely seen in identified DLA absorbers (e.g. Ledoux, Petitjean \& Srianand 2003).

In this regard it is interesting that Gardner et al. (1997) found in their CDM simulations that depletion of the gas supply by star formation only affected the DLA statistics at $z>2$ for $N(H I)>10^{22}$ atoms $\mathrm{cm}^{-2}$ (i.e. in a regime where DLAs have not generally been found), even though roughly half of the cold collapsed gas was converted to stars by $z=2$. But if significant mass has been missed due to small total cross-section for starforming regions, whether or not these high-gas-mass regions will be found once sample sizes are much larger is unclear. Substantial dust-induced reddening may prevent complete samples from ever being discovered via optical quasar absorption line spectroscopy. However, in the end, analysis of intervening absorption line systems in SDSS quasars can still provide powerful constraints on the evolution of metals and the properties of gaseous structures in the Universe.

\section{Acknowledgements}

We thank members of the SDSS collaboration who made the SDSS project a success. We thank Nick Allen and Daniel Owen who helped measure MgII absorption 
lines during their participation in an REU program at the University of Pittsburgh. We also thank Andrew Hopkins for collaborative work and discussions about cosmic SFRs, Jason Prochaska for providing information on DLA velocity spreads for individual DLAs, and Regina Schulte-Ladbeck for discussions about dwarf galaxies. We acknowledge and are grateful for concurrent collaborative work, especially with Brice Ménard, Dan Vanden Berk, and Don York on SDSS MgII absorption spectra. We acknowledge financial support from NASA-STScI, NASA-LTSA, and NSF. HST-UV spectroscopy made the $N(H I)$ determinations possible, while the SDSS spectra made the metallicity measurements possible. Funding for creation and distribution of the SDSS Archive has been provided by the Alfred P. Sloan Foundation, Participating Institutions, NASA, NSF, DOE, the Japanese Monbukagakusho, and the Max Planck Society. The SDSS Web site is www.sdss.org. The SDSS is managed by the Astrophysical Research Consortium for the Participating Institutions: University of Chicago, Fermilab, Institute for Advanced Study, the Japan Participation Group, Johns Hopkins University, Los Alamos National Laboratory, the Max-Planck-Institute for Astronomy (MPIA), the Max-Planck-Institute for Astrophysics (MPA), New Mexico State University, University of Pittsburgh, Princeton University, the United States Naval Observatory, and University of Washington.

\section{References}

Dessauges-Zavadsky, M., Prochaska, J., D’Odorico, S., 2002, A\&A, 391, 801

Edvardsson, B., et al., 1993, A\&A, 275, 101

Gardner, J., Katz, N., Hernquist, L., Weinberg, D., 1997, ApJ, 484, 31

Haehnelt, M., Steinmetz, M., Rauch, M., 1998, ApJ, 495, 647

Hopkins, A., Rao, S., Turnshek, D., 2005, ApJ, in press (astro-ph/0505418)

Kennicutt, R., 1998, ApJ, 498, 541

Ledoux, C., Petitjean, P., Møller, P., Fynbo, J., Srianand, R., 2005, this volume (astro$\mathrm{ph} / 0504402)$

Ledoux, C., Petitjean, P., Srianand, R., 2003, MNRAS, 346, 209

Lu, L., Sargent, W., Barlow, T., Churchill, C., Vogt, S., 1996, ApJS, 107, 475

McWilliam, A., 1997, ARA\&A, 35, 503

Mo, H., Mao, S., White, S., 1998, MNRAS, 295, 319

Mo, H., Mao, S., White, S., 1999, MNRAS, 304, 175

Ménard, B., Nestor, D., Turnshek, D., Richards, G., Rao, S., 2005, ApJ, submitted

Nestor, D., Rao, S., Turnshek, D., Vanden Berk, D., 2003, ApJ, 595, L5

Nestor, D., Turnshek, D., Rao, S., 2005a, ApJ, in press (astro-ph/0410493)

Nestor, D., Turnshek, D., Rao, S., 2005b, this volume (astro-ph/0505134)

Prochaska, J., Gawiser, E., Wolfe, A., Castro, S., Djorgovski, S., 2003, ApJ, 595, L9

Rao, S., 2005, this volume (astro-ph/0505479)

Rao, S., Nestor, D., Turnshek, D., Lane, W., Monier, E., Bergeron, J., 2003, ApJ, 595, 94

Rao, S., Prochaska, J., Howk, C., Wolfe, A., 2005b, AJ, 129, 9

Rao, S., Turnshek, D., 2000, ApJS, 130, 1

Rao, S., Turnshek, D., Nestor, D., 2005a, ApJ, submitted (RTN2005)

Savage, B., Sembach, K., 1996, ARA\&A, 34, 279

Shapley, A., Steidel, C., Pettini, M., Adelberger, K., 2003, ApJ, 588, 65

Umeda, H., Nomoto, K., 2002, ApJ, 565, 385 